\title{
Monitoring the Burden of Seizures and Highly Epileptiform Patterns in Critical Care with a Novel Machine Learning Method
}

\author{
Baharan Kamousi', Suganya Karunakaran' ${ }^{1}$ Kapil Gururangan², Matthew Markert³ ${ }^{3}$ Barbara Decker ${ }^{4}$, \\ Pouya Khankhanian ${ }^{4}$, Laura Mainardi ${ }^{4}$, James Quinn ${ }^{5}$, Raymond Woo ${ }^{1}$ and Josef Parvizi ${ }^{3^{*}}$ (D)
}

(c) 2020 The Author(s)

\begin{abstract}
Introduction: Current electroencephalography (EEG) practice relies on interpretation by expert neurologists, which introduces diagnostic and therapeutic delays that can impact patients' clinical outcomes. As EEG practice expands, these experts are becoming increasingly limited resources. A highly sensitive and specific automated seizure detection system would streamline practice and expedite appropriate management for patients with possible nonconvulsive seizures. We aimed to test the performance of a recently FDA-cleared machine learning method (Clarity, Ceribell Inc.) that measures the burden of seizure activity in real time and generates bedside alerts for possible status epilepticus (SE).
\end{abstract}

Methods: We retrospectively identified adult patients $(n=353)$ who underwent evaluation of possible seizures with Rapid Response EEG system (Rapid-EEG, Ceribell Inc.). Automated detection of seizure activity and seizure burden throughout a recording (calculated as the percentage of ten-second epochs with seizure activity in any 5-min EEG segment) was performed with Clarity, and various thresholds of seizure burden were tested ( $\geq 10 \%$ indicating $\geq 30 \mathrm{~s}$ of seizure activity in the last $5 \mathrm{~min}, \geq 50 \%$ indicating $\geq 2.5 \mathrm{~min}$ of seizure activity, and $\geq 90 \%$ indicating $\geq 4.5 \mathrm{~min}$ of seizure activity and triggering a SE alert). The sensitivity and specificity of Clarity's real-time seizure burden measurements and SE alerts were compared to the majority consensus of at least two expert neurologists.

Results: Majority consensus of neurologists labeled the 353 EEGs as normal or slow activity $(n=249)$, highly epileptiform patterns (HEP, $n=87$ ), or seizures [ $n=17$, nine longer than 5 min (e.g., SE), and eight shorter than $5 \mathrm{~min}$ ]. The algorithm generated a SE alert ( $\geq 90 \%$ seizure burden) with 100\% sensitivity and $93 \%$ specificity. The sensitivity and specificity of various thresholds for seizure burden during EEG recordings for detecting patients with seizures were $100 \%$ and $82 \%$ for $\geq 50 \%$ seizure burden and $88 \%$ and $60 \%$ for $\geq 10 \%$ seizure burden. Of the $179 \mathrm{EEG}$ recordings in which the algorithm detected no seizures, seizures were identified by the expert reviewers in only two cases, indicating a negative predictive value of $99 \%$.

Discussion: Clarity detected SE events with high sensitivity and specificity, and it demonstrated a high negative predictive value for distinguishing nonepileptiform activity from seizure and highly epileptiform activity.

Conclusions: Ruling out seizures accurately in a large proportion of cases can help prevent unnecessary or aggressive over-treatment in critical care settings, where empiric treatment with antiseizure medications is currently

\footnotetext{
*Correspondence: jparvizi@stanford.edu

${ }^{3}$ Department of Neurology and Neurological Sciences, Stanford

University Medical Center, 300 Pasteur Drive, Stanford, CA 94305, USA

Full list of author information is available at the end of the article
}

\section{实 Springer}


prevalent. Clarity's high sensitivity for SE and high negative predictive value for cases without epileptiform activity make it a useful tool for triaging treatment and the need for urgent neurological consultation.

Keywords: Machine learning method, Neurology, Electroencephalography, Status epilepticus, Seizure burden

\section{Introduction}

The timely diagnosis and treatment of patients with seizures can prevent significant morbidity and mortality [1]. Approximately $30 \%$ of patients with altered mental status in critical care settings have seizures, and over $90 \%$ of these are nonconvulsive seizures that can only be detected with electroencephalography (EEG) [2-6]. There is rising awareness among healthcare practitioners that the burden of seizures, including nonconvulsive seizures, is associated with brain injury and, thus, continuous or frequent seizures (i.e., a high seizure burden) merit timely detection and treatment [7-10]. For this reason, both timely interpretation of EEG data and timely and accurate quantification of seizure burden are paramount to minimizing brain injury.

Unfortunately, the conventional practice of EEG in critical care and emergency department settings suffers from both delayed access to EEG recordings and significant delays in its interpretation by skilled neurologists $[11,12]$. When actionable EEG interpretations are not immediately available, treatment decisions are made on the basis of clinical suspicion alone, which results in the potential for missing or undertreating some patients with nonconvulsive seizures and overtreating a significantly larger number of patients without seizure activity who may not need aggressive antiseizure medications $[13,14]$. To provide timely access to EEG, novel rapid EEG systems can be used to enable physicians and allied health professionals to acquire EEG within minutes and stream the data in real time to the cloud, where a machine learning-derived classification algorithm can be applied [15-17].

The use of artificial intelligence (AI) in clinical medicine has been on the rise, and within the specialty of neurology, brain signals have proven particularly amenable to the machine learning approach [18]. There are few software programs commercially available to detect seizures or epileptiform discharges and mark the EEG tracing to help streamline expert review by neurologists. More novel methods for automatic detection of seizures and epileptic spikes have also been described in the literature with various methods and varying degrees of accuracy [19]. Such algorithms are designed to work solely with traditional EEG systems, which as noted are often delayed or unavailable, especially during after-hours and weekends $[11,12]$ and are too cumbersome for nonneurology experts to use at the bedside. While a full review of these methods is beyond the scope of our present work, it is noteworthy that no method has yet been developed to provide an automated and quantified metric of seizure burden (i.e., frequency of seizures per unit of time) to help bedside practitioners caring for critically ill patients. Providing such feedback would allow for risk stratification and evaluation of treatment response, as well as for determining the urgency of requesting neurological consultation, in real time.

The use of AI-assisted programs for EEG interpretation is becoming increasingly necessary as the utilization of EEG is expanding in the fields of critical care and emergency medicine while human resources are scarce, and detailed review of many simultaneous continuous EEG recordings by neurologists in real time is simply too cost prohibitive to be deployed at scale. As a result, there exists a significant unmet need for automated algorithms that could assist nonexperts by providing a reliable risk stratification tool using EEG data in real time [18, 20, 21]. Such a tool could alert the bedside nurse or provider on call when it detects a near-continuous epileptiform pattern resembling status epilepticus that may require urgent management and enable providers to see the realtime effect of administered antiseizure medications on the burden of seizure activity.

In the current study, we aim to validate a supervised machine learning algorithm, labeled as Clarity (Ceribell Inc., Mountain View, California), that was recently approved by the FDA and is being used in clinical settings as a clinical decision support tool. We designed this retrospective study to measure the performance of the algorithm applied to EEG data acquired using Ceribell's Rapid Response EEG system (Rapid-EEG) from patients in critical care and emergency department settings and to describe its potential clinical implications.

\section{Methods}

\section{Rapid Response EEG System}

The Rapid-EEG system (Fig. 1, see www.ceribell.com for additional information) consists of a headband with ten electrodes connected to a handheld recorder. The headband is placed circumferentially around the head and is fastened over the forehead with a locking clasp, and EEG setup is typically performed by nurses, allied health professionals, or healthcare providers trained by either online or in-person sessions. The Rapid-EEG electrodes (1-5 on the left, $6-10$ on the right; electrode number increases anterior to posterior, i.e., leads 1 and 6 


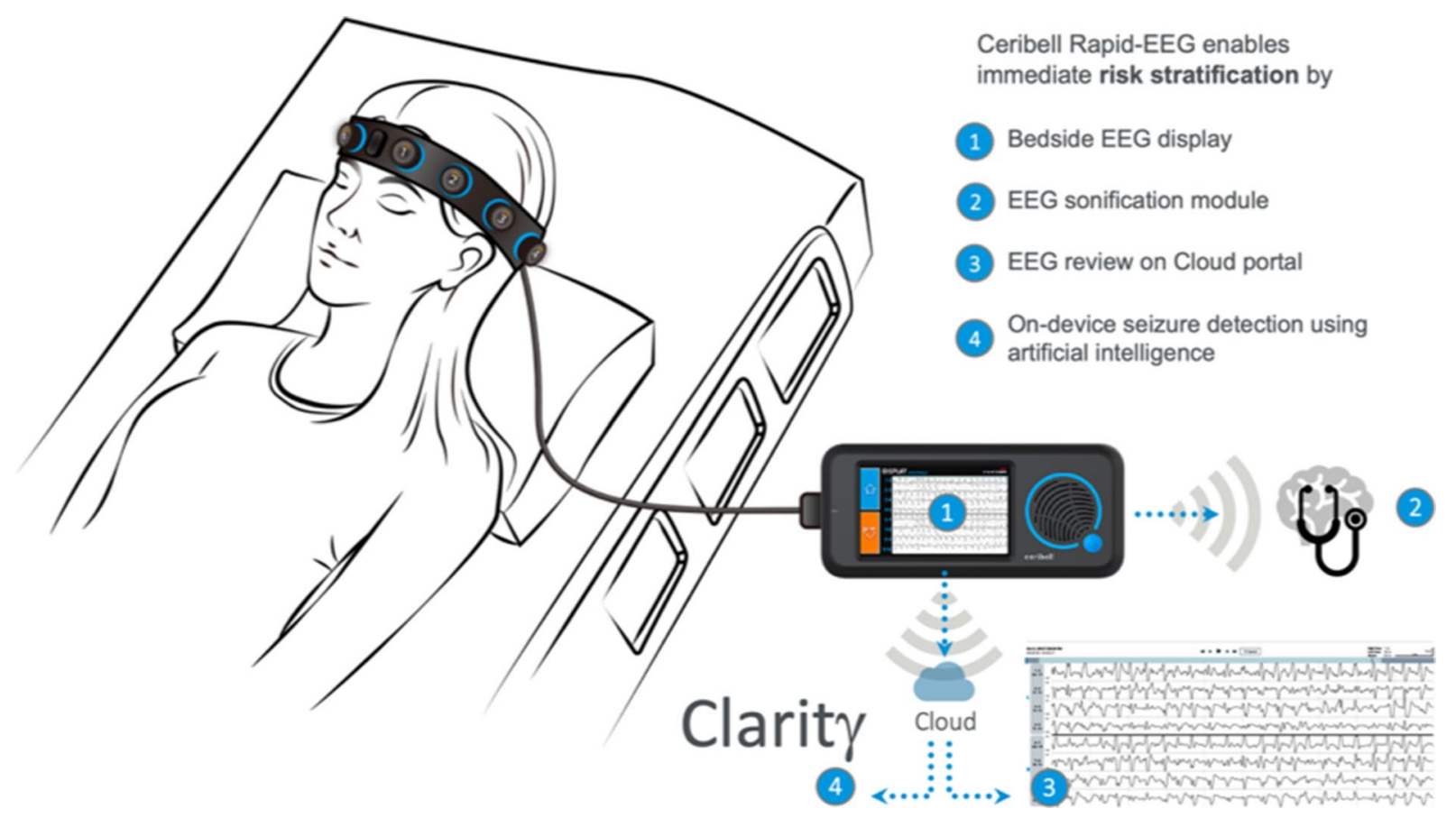

Fig. 1 Rapid Response EEG system. The Rapid Response EEG system (Rapid-EEG) consists of a portable EEG recorder and a disposable electrode headband. Recorded EEG tracings are shown on the device screen (1) and sonified when needed (2) by the bedside recorder. HIPAA-compliant secure Wi-Fi connection enables real-time transfer of the data to the cloud where the EEG tracings can be reviewed by expert neurologists using the remote portal for EEG review (3). Machine learning computations (by Clarity algorithm) are performed on the cloud portal (4) interfacing in real time with the bedside device. As such, the system is meant to provide not only easy and fast access to EEG acquisition, but also a reliable and actionable diagnostic information for risk stratification using four different modes of triage

are near the frontal pole and leads 5 and 10 are near the occiput) correspond approximately to the lateral chains of the International 10-20 system (Fp1-F7, F7-T3, T3T5, and T5-O1 on the left; Fp2-F8, F8-T4, T4-T6, and T6-O2 on the right), and a longitudinal bipolar montage is constructed to display the EEG waveforms. During EEG recording, the system simultaneously measures the impedance between two adjacent electrodes once per minute using a test frequency that is outside the EEG recording band to avoid creating artifact. The data are acquired as digital samples at a rate of $250 \mathrm{~Hz}$. The handheld recorder displays, records, and wirelessly transmits the data to a remote cloud server, where a cloud-based seizure detection software continuously monitors the EEG recording.

\section{EEG Data}

All EEGs were obtained with Rapid-EEG (Ceribell Inc., Mountain View, California). The recordings were from adult patients ( $\geq 18$ years old) undergoing evaluation for altered mental status and possible seizures in intensive care units and emergency departments at six academic and community hospitals across the USA between January 2018 and April 2019. EEG data were anonymized, and no identifying demographic or clinical information was accessed for this study. The study was classified as exempt research according to the US Department of Health and Human Services regulation 45 CFR 46.104(d) (4), and individual patient consent was not required. It should be noted that the Clarity algorithm was developed using training and testing datasets that were entirely different and independent from the dataset we have used in the current study for the validation of the algorithm performance.

\section{Validating Clarity Seizure Burden Algorithm}

We validated the performance of the Clarity algorithm in a cohort of 353 Rapid-EEG recordings from 353 patients. An overview of the algorithm is shown in Fig. 2. The signal from each EEG channel was filtered and segmented into nonoverlapping ten-second bins. Time-domain and frequency-domain features were calculated for each tensecond bin of EEG signal. Seizure activity was defined for each ten-second bin using multiple features, including measures of power (power within each frequency band and their ratios), morphology (signal amplitude, variability, distribution, and change over time), rhythmicity and regularity (measures of entropy), and correlation 


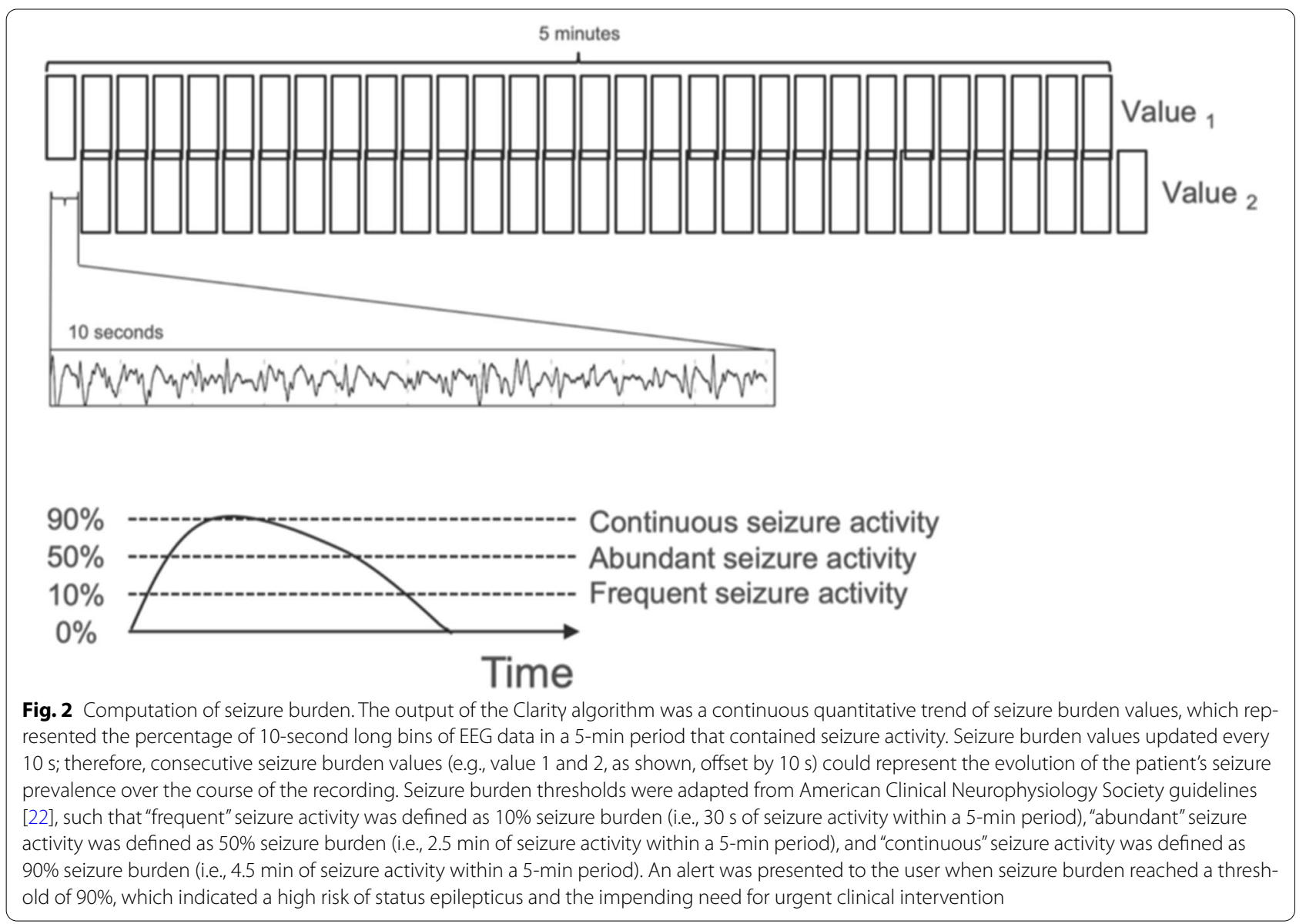

(cross-channel correlation of signals). For each ten-second bin, the algorithm classified the segment of signal as either seizure or nonseizure in a deterministic (rather than probabilistic) manner. Seizure burden was calculated as the percentage of ten-second bins of EEG data in a 5 -min period that were classified as seizure activity. This seizure burden value is updated every ten seconds to generate a rolling 5-min window, resulting in a continuous seizure burden trend that represents the evolution of the patient's seizure prevalence over the course of monitoring. The maximum seizure burden for the duration of each recording was used as the final output of the algorithm for this study; however in clinical practice, the seizure burden value and trend would be available continuously.

Seizure burden, the prevalence of seizure activity within any 5-min period, was described using thresholds adapted from American Clinical Neurophysiology Society guidelines [22] as follows: $10 \%$ was defined as "frequent" (indicating $30 \mathrm{~s}$ of seizure activity), 50\% was defined as "abundant" (indicating 2.5 min of seizure activity), and 90\% was defined as "continuous" (indicating $4.5 \mathrm{~min}$ of seizure activity). A seizure burden of $90 \%$
(4.5 min) indicates activity approaching the definition of status epilepticus ( $5 \mathrm{~min}$ ), so the algorithm would present an alert to the user at any point when the seizure burden reaches a threshold of $90 \%$.

\section{Reference Standard Defined by Expert Neurologists' Review of EEG}

Each EEG file was independently reviewed by at least two independent neurologists with fellowship training in clinical neurophysiology or epilepsy. Reviewers were blinded to patients' clinical information, including medical history, indication for EEG monitoring, prior treatment with antiseizure medication, and Clarity seizure burden trend. Expert consensus (reference standard) was defined by agreement between a minimum of two neurologists; additional reviewers were consulted if the first two neurologists did not agree until a majority consensus was reached. Reviewers were instructed to indicate whether the EEG contained normal, diffusely slow, highly epileptiform, or seizure activity. Highly epileptiform patterns (HEP) included activity that did not fully meet the Salzburg criteria [23] for electrographic seizure activity, but did represent abnormal electrographic epileptiform 
activity such as periodic discharges or lateralized rhythmic delta activity [24, 25]. These patterns merited separate consideration from other nonseizure patterns for two major reasons. First, these patterns exist on a spectrum of pathologic activity along with seizures-the socalled ictal-interictal continuum (IIC)-and at times, they can be difficult to distinguish from seizures, especially absent ancillary clinical information [26-28]. Second, these patterns might benefit from treatment with antiseizure medications, and measuring the performance of the algorithm in these cases would have clinical significance [26, 29-31]. See Fig. 3 for a representative sample of each of these categories recorded using Rapid-EEG. Each EEG recording and each individual labeled episode were divided into one of three categories based on the most severe pattern present in the recording defined by expert majority consensus: seizures (high severity), HEP (intermediate severity), and normal or slow activity (low severity). Therefore, a reference standard was generated for individual episodes as well as for the overall EEG recording. EEG readers also labeled the start and end of the EEG patterns, which allowed for calculation of seizure duration that could be compared to the algorithm output.

\section{Statistical Analysis}

We defined the reference ("gold") standard as the consensus agreement of at least two neurologists reading the same
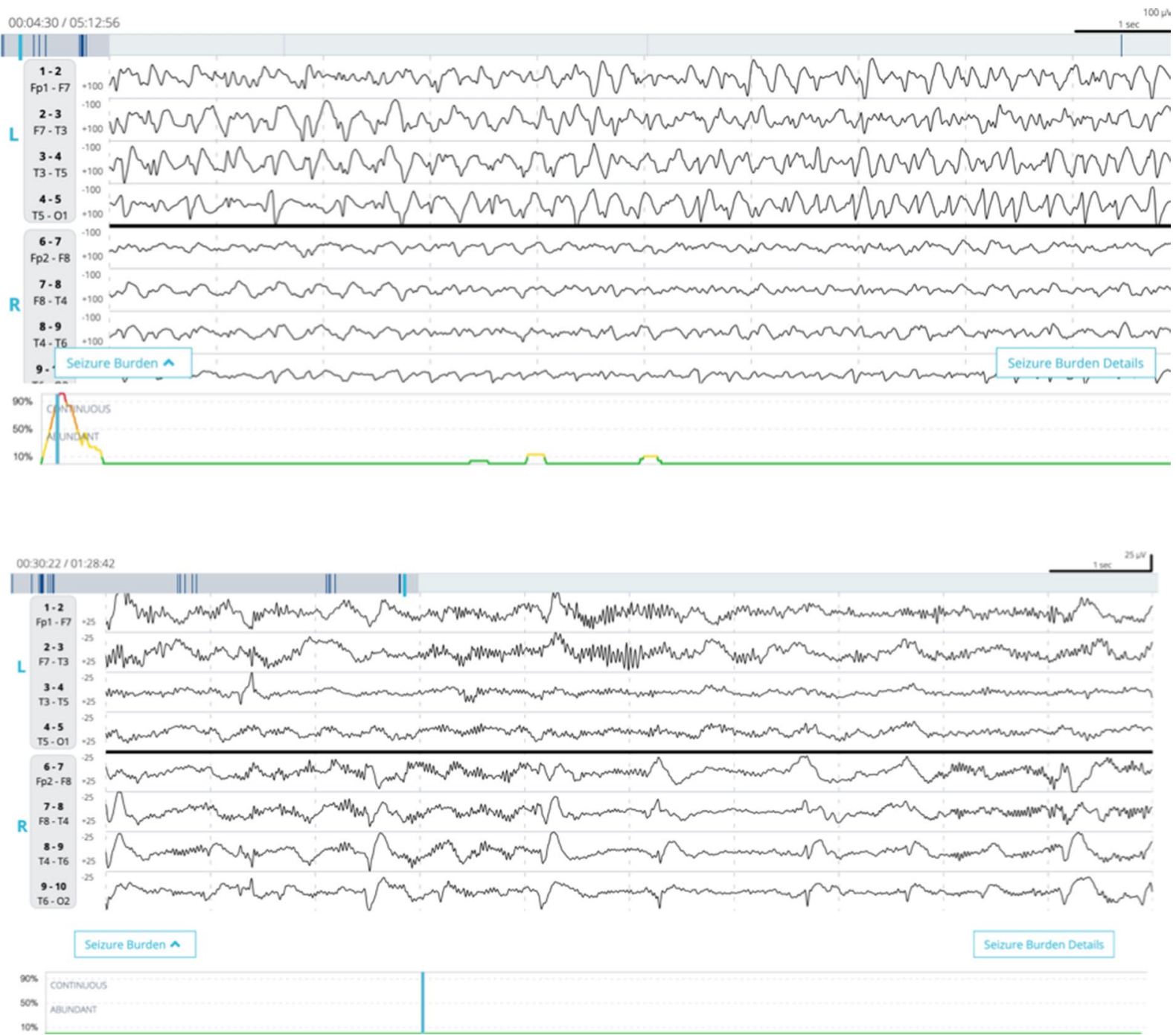

Fig. 3 Samples of EEG recorded with Ceribell Rapid Response EEG System. Each EEG is displayed in a ten-second epoch with filter settings of 1-30 Hz. The line plot under each EEG shows the Clarity algorithm output. The top image shows seizure activity approaching the $90 \%$ threshold to trigger a status epilepticus alert, and the bottom image shows lateralized periodic discharges that go undetected by the algorithm 
EEG, both for each overall EEG record and for individual episodes of expert-identified seizures. We then tabulated Clarity algorithm output of seizure burden against this reference standard. Using these tabulations, we calculated the sensitivity, specificity, and false detection rate (number of false positive events divided by the total duration of EEG recordings in hours) of various seizure burden thresholds $(10 \%, 50 \%, 90 \%)$ against the expert consensus; $95 \%$ CIs for sensitivity and specificity measures were calculated using established formulas [32]. It should be noted that the three thresholds are cumulative-i.e., all $90 \%$ alerts by definition generate both a $10 \%$ and a $50 \%$ notification, and all $50 \%$ alerts by definition first generate a $10 \%$ notification. Our validation study was not designed to optimize among the three different thresholds.

Given the wealth of evidence [33-36] describing the variability between expert EEG reviewers, whether due to human error or to differences in interpretation, we sought to contextualize the observed diagnostic accuracy of the seizure detection algorithm by quantifying the inter-rater variability. We calculated the sensitivity and specificity of identifying EEGs with status epilepticus for each of the experts that reviewed at least $250 \mathrm{~h}$ of EEG. No single expert reviewed all of the cases, and because each of the experts reviewed a different subset of the recordings, we could not quantify inter-rater variability using Cohen's $\kappa$.

\section{Results}

\section{Reference Standard}

A total of 353 Rapid-EEG recordings were evaluated in this study with a cumulative EEG recording duration of $1052 \mathrm{~h}$ (mean EEG duration: $233 \pm 227 \mathrm{~min}$ ). No cases were excluded. Expert consensus determined the most severe feature of the overall EEG record to be status epilepticus in nine cases, seizures (duration $<4.5 \mathrm{~min}$ ) in eight cases, HEP in 87 cases, and normal or low background in 249 cases (Table 1). Within the 353 recordings, a total of 47 discrete seizure events were identified by expert consensus.

\section{Algorithm Performance}

Clarity algorithm output of seizure burden compared against expert consensus diagnosis for both overall EEG records and individual events is summarized in Table 1, and performance (in terms of sensitivity, specificity, and false detection rate) is summarized in Table 2. Clarity detected $\geq 90 \%$ seizure burden (seizure activity $\geq 4.5 \mathrm{~min}$, thereby triggering an alert for impending status epilepticus) in nine out of nine Rapid-EEGs with status epilepticus, and within these cases, Clarity correctly detected $\geq 90 \%$ seizure burden in 12 out of 13 discrete events of seizure lasting $\geq 4.5 \mathrm{~min}$. Therefore, the sensitivity for identifying status epilepticus was $100 \%$ for

\begin{tabular}{lllllll}
$\begin{array}{l}\text { Table } 1 \text { Summary } \\
\text { patient level) }\end{array}$ & of & Clarity & Performance & (individual \\
$\begin{array}{l}\text { Clarity output, } \% \\
\text { seizure burden }\end{array}$ & \multicolumn{5}{l}{ Human expert rating, $n$} \\
\cline { 2 - 6 } & SE & SZ & HEP & NL/SL & Total \\
\hline SZ burden $\geq 90 \%$ & 9 & 0 & 21 & 3 & 33 \\
\hline SZ burden 50-89\% & 0 & 3 & 21 & 15 & 39 \\
\hline SZ burden 10-49\% & 0 & 3 & 20 & 56 & 79 \\
SZ burden 1-9\% & 0 & 0 & 5 & 18 & 23 \\
\hline SZ burden 0\% & 0 & 2 & 20 & 157 & 179 \\
\hline Total & 9 & 8 & 87 & 249 & 353 \\
\hline
\end{tabular}

HEP highly epileptiform pattern, NL normal background activity, SE status epilepticus, SL slow background activity, SZ seizure

the overall record, and the sensitivity for identifying individual seizure episodes that were $\geq 4.5$ min was $92.3 \%$.

The specificity of the $\geq 90 \%$ seizure burden notification was $93.0 \%$, resulting in a false detection for only 24 out of 353 EEGs. In 21 (87.5\%) of these false detections, the expert consensus categorization of the EEG was HEP, indicating that there was concerning epileptiform activity present in the record, even if no unequivocal seizures were agreed to be present by both reviewers.

Clarity correctly identified 41 of the 47 total discrete seizure events of any duration ( $88.2 \%$ sensitivity). In the $1052 \mathrm{~h}$ of cumulative EEG across the 353 EEG recordings, Clarity had 383 false detections, resulting in an overall false detection rate of 0.36 per hour. Of the 179 EEG recordings in which Clarity detected no seizures, seizures were identified by the expert reviewers in only two cases (negative predictive value of 99\%). In both cases, the seizures missed by Clarity were less than $30 \mathrm{~s}$ in duration.

The four experts reviewed at least $250 \mathrm{~h}$ of the overall dataset (1052 total hours). When these four reviewers' identifications of status epilepticus were compared to the majority consensus (Table 3), individual expert raters displayed variability in both sensitivity (range $20-89 \%$ ) and specificity (range 94-99\%). In general, reviewers with the lowest sensitivity also had the highest specificity, a natural trade-off. It is also important to note that, in some cases, reviewers classified a status epilepticus case as a highly epileptiform pattern (HEP). This was considered a miss and reduced the reviewer's sensitivity even though the reviewer did not consider the EEG to be normal. Each expert reviewed a different subset of the 353 total recordings. Between the four experts, a total of 784 individual reviews were performed (an average of 2.22 reviews per EEG recording). No single expert reviewed all of the cases. The number of reviews performed by each expert is shown in Table 3. Overall, experts displayed greater specificity compared to Clarity, and each of the reviewers misclassified at least one case of status epilepticus. 
Table 2 Sensitivity and specificity of Clarity algorithm for seizure detection

\begin{tabular}{|c|c|c|c|c|c|c|c|}
\hline \multirow[t]{2}{*}{ Clarity output } & \multicolumn{3}{|c|}{ Patient level } & \multicolumn{4}{|c|}{ Event level } \\
\hline & $N$ & Sensitivity $(95 \% \mathrm{CI})$ & $\begin{array}{l}\text { Specificity } \\
(95 \% \text { CI) }\end{array}$ & $N$ & Sensitivity $(95 \% \mathrm{CI})$ & $\begin{array}{l}\text { False positives } \\
(95 \% \mathrm{Cl})\end{array}$ & FDR $^{a}$ \\
\hline SZ burden $\geq 90 \%$ & 9 & $100.0 \%^{c}$ & $93.0 \%$ & 13 & $92.3 \%{ }^{\mathrm{b}}$ & 62 & 0.06 \\
\hline $95 \% \mathrm{Cl}$ & & & {$[90,95]$} & & {$[60,100]$} & & \\
\hline SZ burden $\geq 50 \%$ & 12 & $100.0 \%{ }^{c}$ & $82.4 \%$ & 18 & $100.0 \%{ }^{c}$ & 139 & 0.13 \\
\hline $95 \% \mathrm{Cl}$ & & & {$[78,87]$} & & & & \\
\hline SZ burden $\geq 10 \%$ & 17 & $88.2 \%$ & $59.5 \%$ & 35 & $80.0 \%$ & 324 & 0.31 \\
\hline $95 \% \mathrm{Cl}$ & & {$[65,100]$} & {$[54,65]$} & & {$[63,91]$} & & \\
\hline
\end{tabular}

$\mathrm{Cl}$ confidence interval, FDR false detection rate

${ }^{a}$ False detection rate (in events per hour of EEG) was calculated as the number of false positive events divided by the duration of recording (in hours)

${ }^{b}$ One seizure event that did not trigger a status alarm occurred during the last 10 min of a 200-min EEG record. The algorithm correctly identified the seizure, but the threshold for $90 \%$ seizure burden $(4.5 \mathrm{~min}$ ) was not yet reached at the time the recording was discontinued

${ }^{c}$ Confidence intervals are not calculated in cases where the sampled sensitivity was $100 \%$ as estimated confidence intervals in the event of perfect sample sensitivity do not provide meaningful information

Table 3 Variability in status epilepticus detection between individual experts and Clarity status alert compared to expert consensus

\begin{tabular}{llll}
\hline Reviewer & $\begin{array}{l}\text { Sessions } \\
\text { reviewed }\end{array}$ & Sensitivity (\%) & Specificity (\%) \\
\hline 1 & 240 & 62.5 & 94.8 \\
2 & 198 & 20.0 & 99.5 \\
3 & 257 & 88.9 & 94.3 \\
4 & 89 & 66.7 & 95.2 \\
Clarity & 353 & 100.0 & 93.0 \\
\hline
\end{tabular}

\section{Discussion}

In this study, we describe the development of an artificial intelligence machine learning algorithm for seizure burden measurement using EEG data acquired with Rapid Response EEG. This algorithm showed high sensitivity $(100 \%)$ in the detection of status epilepticus (even outperforming some neurologists with fellowship training in clinical neurophysiology or epilepsy) and accurately identified $88 \%$ of seizures of any duration when compared to the reference standard of consensus of expert neurologists (Fig. 4). The specificity of the algorithm for seizures at all seizure burden thresholds was principally limited by "misclassification" of highly epileptiform patterns. These patterns possess multiple "seizure-like" qualities trained into our algorithm (e.g., rhythmicity, sharply contoured morphology, high amplitude, and extant correlation across channels) as seizures. Raising the seizure burden threshold to trigger an alarm (i.e., only alerting the user if the burden reaches $90 \%$ level) increased the algorithm's specificity for prolonged seizures as high as $93 \%$. As such, the algorithm performance can be considered to be reliable and valid at the extremes of the spectrum of seizure burden (i.e., possible status epilepticus and normal diffusely slow activity), but considerably less so if the burden of abnormality is less frequent or brief in duration. While our new algorithm output provides a trendline and a means to assist in triage/prioritization, it is important for users to understand its lower specificity for milder cases of abnormality or shorter duration seizures.

\section{Suggested Clinical Implementation Workflow}

In considering the potential clinical implications of Rapid Response EEG with Clarity, it is important to ensure its proper integration into existing workflows to secure its maximum (and safe) impact on physicians' clinical decision making and patient management. We remind the reader that the output of any AI algorithm ought to be interpreted in the context of the user's pretest clinical judgment. In keeping with this, we have made an initial attempt to recommend a possible workflow for the use of our algorithm in the current clinical practice of RapidEEG (Table 4). We are mindful that the workflow may need to be modified in different settings depending on the resources available and the clinical division where the Rapid-EEG technology is being utilized.

As suggested in this workflow, Clarity algorithm has potential utility as the first of its kind risk stratification tool to streamline the practice of stat EEG and guide emergent triage and more precise treatment for patients with low or high suspicion for nonconvulsive seizures. Moreover, it also can lessen the stress and unnecessary burden on neurologists with EEG expertise. We are hopeful that simplified risk stratification offered by our algorithm combined with the earlier and easier acquisition of EEG will lead to better management of patients in need. 


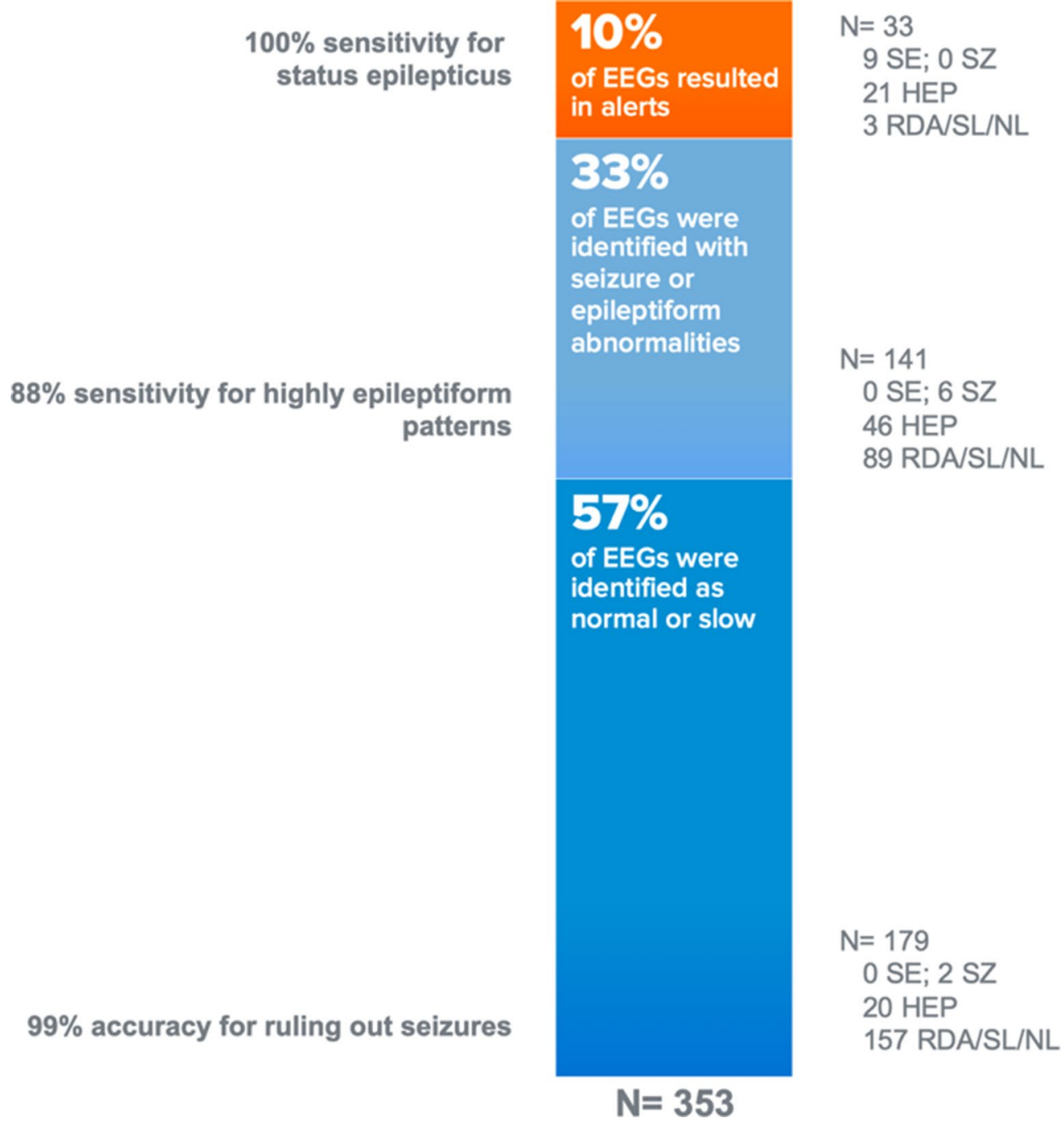

Fig. 4 Summary of Clarity Performance. Performance of Clarity algorithm at the group level suggests that the algorithm can be seen as a reliable triage tool to help detect cases of status epilepticus with the highest sensitivity (while overcalling about one-fourth of highly epileptiform patterns as possible status epilepticus). It also performs as a reliable triage tool to help physicians avoid over-aggressive treatments in majority of EEG cases where the overwhelming pattern is either slowing or normal. HEP highly epileptiform patterns, NL normal activity, RDA rhythmic delta activity, SE status epilepticus, SL slow activity, SZ seizure

\section{Table 4 Suggested clinical implementation workflow of Clarity algorithm}

\begin{tabular}{|c|c|c|c|}
\hline & & \multicolumn{2}{|l|}{ Pretest clinical suspicion } \\
\hline & & High & Low \\
\hline \multirow[t]{3}{*}{ Al output } & $>90 \%$ & Treat urgently $\rightarrow$ urgent review of EEG ${ }^{a}$ & $\begin{array}{l}\text { Review of EEG } \rightarrow \text { treat if seizures confirmed on EEG or if } \\
\text { EEG reading is not readily possible }\end{array}$ \\
\hline & $10-90 \%$ & $\begin{array}{l}\text { Review of EEG } \rightarrow \text { treat if seizures confirmed on EEG or if EEG } \\
\text { reading is not readily possible }\end{array}$ & Do not treat yet $\rightarrow$ Review of EEG whenever possible \\
\hline & $<10 \%$ & Do not treat yet $\rightarrow$ Review of EEG whenever possible & Do not treat $\rightarrow$ nonurgent review of EEG \\
\hline
\end{tabular}

a Expedited review of EEG can be done remotely in real time by a neurologist with EEG expertise since the Rapid-EEG device sends the EEG data wirelessly to a cloud portal. EEG review can also be performed at the bedside by both expert or nonexpert users. For instance, similar to common models of electrocardiographic monitoring [40] or bedside quantitative EEG products [41,42], critical care staff may be trained to recognize the most salient and clinically important EEG signatures associated with status epilepticus. They can rely on their own bedside visual EEG review combined with the Rapid-EEG's Brain Stethoscope function [43]. In three clinical studies so far, staff with minimal or no EEG experience increased their accuracy of seizure diagnosis significantly by relying on either Brain Stethoscope alone $[15,16]$ or combined with bedside visual EEG review [17]. It is important to note that a much larger number of EEG cases often do not result in seizure output, and hence, use of the algorithm will lead to prevention of overtreatment of these cases. Given the high sensitivity of the algorithm, false negative cases would be much less frequent 


\section{Study Limitations}

The lack of clinical data provided to reviewers during their retrospective EEG review was the most important limitation of this study and should be considered highly relevant to the interpretation of its findings. In clinical practice, patient history (e.g., an established diagnosis of epilepsy with a known seizure semiology, recent antiseizure medications) and bedside or video observations (e.g., twitching or behavioral changes) are used to inform EEG interpretation and make definitive determinations of seizure versus nonseizure, and treatment is tailored to the patient as a whole rather than the EEG in isolation. Clarity, like all other automated seizure detection algorithms, does not consider ancillary clinical information, and it is critical to acknowledge that output from such an algorithm does not provide the final diagnostic conclusion for the patient. Indeed, the sensitivity and specificity of any system for EEG review (human or machine) would be significantly improved by access to additional patient data.

The implementation of Clarity and its impact on realworld diagnostic and treatment decisions were not studied here and may be addressed in future investigations. As noted in the discussion of clinical workflow, individual providers or practice groups may utilize different alarm thresholds to expedite EEG review and treatment or establish treatment protocols that rely on the various functions of Rapid-EEG (visual waveform review, EEG sonification, Clarity seizure burden) based on providers' comfort with each.

Our cohort contained a relatively low number of status epilepticus cases (nine out of 353 EEGs) which affects the reliability of our sensitivity estimate. A larger cohort of Rapid-EEG data would be helpful to address this. However, it should be noted that this multisite cohort was obtained without excluding cases and could be considered reflective of the population being studied.

The Rapid-EEG system lacks midline and parasagittal electrodes, and consequently a focal seizure that is highly localized to the parasagittal region would not be detected by Rapid-EEG or identified by Clarity (although prior research [37-39] has argued that this lack of coverage might not significantly impact its sensitivity in critical care and emergency medicine settings).

\section{Conclusions}

Implementation of artificial intelligence tools in the field of neurology and epileptology remains limited despite the urgent need for tools to accommodate the expanding practice of EEG and address the inefficiencies of the current EEG infrastructure. Clarity provides highly sensitive detection of status epilepticus and may be useful as a risk stratification tool that could expedite diagnosis and treatment of patients with nonconvulsive seizures. We believe that machine learning tools will never replace a careful history and examination filtered through a well-honed clinical acumen of the user and recommend its safe use by interpreting its output in the context of proper clinical judgment.

\begin{abstract}
Author details
${ }^{1}$ Ceribell Inc., 2483 Old Middlefield Way, Suite 120, Mountain View, CA, USA. ${ }^{2}$ Department of Neurology, The Mount Sinai Hospital, New York, NY, USA. ${ }^{3}$ Department of Neurology and Neurological Sciences, Stanford University Medical Center, 300 Pasteur Drive, Stanford, CA 94305, USA. ${ }^{4}$ Department of Neurology, Perelman School of Medicine, University of Pennsylvania, Philadelphia, PA, USA. ${ }^{5}$ Department of Emergency Medicine, Stanford University Medical Center, Stanford, CA, USA.
\end{abstract}

\section{Acknowledgements}

We thank Drs. Lawrence Hirsch (Yale University) and Eric Rosenthal (Harvard University) for their feedback on the data. Dr. Hirsch serves as scientific advisor to Ceribell. Dr. Rosenthal does not have any conflicts of interest with Ceribell.

\section{Author contributions}

We confirm that all authors have met requirements for authorship. BK, SK, RW, and JP were involved in the concept and design of the study. KG, MM, BD, PK, and LM were involved with data analysis. All authors contributed to drafting and revision of the manuscript and approved the final manuscript.

\section{Source of support}

Funding was provided by Ceribell, Inc.

\section{Conflict of interest}

Drs. Decker, Khankhanian, and Mainardi have no conflicts of interest to declare. Drs. Kamousi, Karunakaran and Woo are members of research team at Ceribell (Mountain View, California) and developed the seizure burden algorithm. Drs. Gururangan, Markert, and Quinn serve as scientific advisers to Ceribell. Dr. Parvizi is main inventor of the EEG system discussed in this manuscript and co-founder of Ceribell. Dr. Quinn's and Dr. Parvizi's contributions to this publication were not part of their Stanford University duties or responsibilities.

\section{Ethical approval and informed consent}

The study was classified as exempt research according to the US Department of Health and Human Services regulation 45 CFR 46.104(d)(4), and individual patient consent was not required.

\section{Open Access}

This article is licensed under a Creative Commons Attribution 4.0 International License, which permits use, sharing, adaptation, distribution and reproduction in any medium or format, as long as you give appropriate credit to the original author(s) and the source, provide a link to the Creative Commons licence, and indicate if changes were made. The images or other third party material in this article are included in the article's Creative Commons licence, unless indicated otherwise in a credit line to the material. If material is not included in the article's Creative Commons licence and your intended use is not permitted by statutory regulation or exceeds the permitted use, you will need to obtain permission directly from the copyright holder. To view a copy of this licence, visit http://creativecommons.org/licenses/by/4.0/.

\section{Publisher's Note}

Springer Nature remains neutral with regard to jurisdictional claims in published maps and institutional affiliations.

Received: 30 May 2020 Accepted: 17 September 2020

Published online: 6 October 2020 


\section{References}

1. Betjemann JP, Lowenstein DH. Status epilepticus in adults. Lancet Neurol. 2015;14(6):615-24.

2. Kaplan PW. Nonconvulsive status epilepticus in the emergency room. Epilepsia. 1996;37(7):643-50.

3. Claassen J, Mayer SA, Kowalski RG, Emerson RG, Hirsch LJ. Detection of electrographic seizures with continuous EEG monitoring in critically ill patients. Neurology. 2004;62(10):1743-8.

4. Claassen J, Jetté N, Chum F, Green R, Schmidt M, Choi H, Jirsch J, Frontera JA, Connolly ES, Emerson RG, et al. Electrographic seizures and periodic discharges after intracerebral hemorrhage. Neurology. 2007:69(13):1356-65.

5. Rossetti AO, Logroscino G, Liaudet L, Ruffieux C, Ribordy V, Schaller MD, Despland PA, Oddo M. Status epilepticus: an independent outcome predictor after cerebral anoxia. Neurology. 2007;69(3):255-60.

6. Varelas PN, Spanaki MV, Mirski MA. Status epilepticus: an update. Curr Neurol Neurosci Rep. 2013;13(7):357

7. Payne ET, Zhao XY, Frndova H, McBain K, Sharma R, Hutchison JS, Hahn CD. Seizure burden is independently associated with short term outcome in critically ill children. Brain. 2014;137(5):1429-38.

8. Claassen J, Albers D, Schmidt JM, De Marchis GM, Pugin D, Falo CM, Mayer SA, Cremers S, Agarwal S, Elkind MS, et al. Nonconvulsive seizures in subarachnoid hemorrhage link inflammation and outcome. Ann Neurol. 2014;75(5):771-81.

9. Vespa P, Tubi M, Claassen J, Buitrago-Blanco M, McArthur D, Velazquez AG, Tu B, Prins M, Nuwer M. Metabolic crisis occurs with seizures and periodic discharges after brain trauma. Ann Neurol. 2016;79(4):579-90.

10. Wagenman KL, Blake TP, Sanchez SM, Schultheis MT, Radcliffe J, Berg RA, Dlugos DJ, Topjian AA, Abend NS. Electrographic status epilepticus and long-term outcome in critically ill children. Neurology. 2014:82(5):396-404

11. Quigg M, Shneker B, Domer P. Current practice in administration and clinical criteria of emergent EEG. J Clin Neurophysiol. 2001;18(2):162-5.

12. Gururangan K, Razavi B, Parvizi J. Utility of electroencephalography: experience from a U.S. tertiary care medical center. Clin Neurophysiol. 2016;127(10):3335-40.

13. Herman ST, Abend NS, Bleck TP, Chapman KE, Drislane FW, Emerson RG, Gerard EE, Hahn CD, Husain AM, Kaplan PW, et al. Consensus statement on continuous EEG in critically ill adults and children, part I: indications. J Clin Neurophysiol. 2015;32(2):87-95.

14. Claassen J, Taccone FS, Horn P, Holtkamp M, Stocchetti N, Oddo M. Recommendations on the use of EEG monitoring in critically ill patients: consensus statement from the neurointensive care section of the ESICM. Intensive Care Med. 2013;39(8):1337-51.

15. Hobbs K, Krishnamohan P, Legault C, Goodman S, Parvizi J, Gururangan K, Mlynash M. Rapid bedside evaluation of seizures in the ICU by listening to the sound of brainwaves: a prospective observational clinical trial of Ceribell's brain stethoscope function. Neurocrit Care. 2018;29(2):302-12.

16. Yazbeck M, Sra P, Parvizi J. Rapid response electroencephalography for urgent evaluation of patients in community hospital intensive care practice. J Neurosci Nurs. 2019:51(6):308-12.

17. Vespa PM, Olson DM, John S, Hobbs KS, Gururangan K, Nie K, Desai MJ, Markert M, Parvizi J, BleckTP, et al. Evaluating the clinical impact of rapid response electroencephalography: the DECIDE multicenter prospective observational clinical study. Crit Care Med. 2020;9:1249-57.

18. Patel UK, Anwar A, Saleem S, Malik P, Rasul B, Patel K, Yao R, Seshadri A, Yousufuddin M, Arumaithurai K. Artificial intelligence as an emerging technology in the current care of neurological disorders. J Neurol (in press).

19. Abbasi B, Goldenholz DM. Machine learning applications in epilepsy. Epilepsia. 2019;60(10):2037-47.

20. Jing J, Sun H, Kim JA, Herlopian A, Karakis I, Ng M, Halford JJ, Maus D, Chan F, Dolatshahi M, et al. Development of expert-level automated detection of epileptiform discharges during electroencephalogram interpretation. JAMA Neurol. 2020;77(1):103-8.

21. Struck AF, Rodriguez-Ruiz AA, Osman G, Gilmore EJ, Haider HA, Dhakar MB, Schrettner M, Lee JW, Gaspard N, Hirsch LJ, et al. Comparison of machine learning models for seizure prediction in hospitalized patients. Ann Clin Transl Neurol. 2019:6(7):1239-47.
22. Hirsch $\sqcup$, LaRoche SM, Gaspard N, Gerard E, Svoronos A, Herman ST, Mani R, Arif H, Jette N, Minazad Y, et al. American clinical neurophysiology society's standardized critical care EEG terminology: 2012 version. J Clin Neurophysiol. 2013;30(1):1-27.

23. Beniczky S, Hirsch LJ, Kaplan PW, Pressler R, Bauer G, Aurlien H, Brøgger JC, Trinka E. Unified EEG terminology and criteria for nonconvulsive status epilepticus. Epilepsia. 2013;54(Suppl 6):28-9.

24. Lewis DW, Johnson EL. Prognosis of periodic and rhythmic patterns in adult and pediatric populations. J Clin Neurophysiol. 2018;35(4):303-8.

25. Maciel $C B$, Hirsch LJ. Definition and classification of periodic and rhythmic patterns. J Clin Neurophysiol. 2018;35(3):179-88.

26. Chong DJ, Hirsch LJ. Which EEG patterns warrant treatment in the critically ill? Reviewing the evidence for treatment of periodic epileptiform discharges and related patterns. J Clin Neurophysiol. 2005;22(2):79-91.

27. Johnson EL, Kaplan PW. Population of the ictal-interictal zone: the significance of periodic and rhythmic activity. Clin Neurophysiol Pract. 2017;2:107-18

28. Kalamangalam GP, Pohlmann-Eden B. Ictal-interictal continuum. J Clin Neurophysiol. 2018;35(4):274-8.

29. Rubinos C, Reynolds AS, Claassen J. The ictal-interictal continuum: to treat or not to treat (and how)? Neurocrit Care. 2018;29(1):3-8.

30. Kapinos G, Trinka E, Kaplan PW. Multimodal approach to decision to treat critically ill patients with periodic or rhythmic patterns using an ictal-interictal continuum spectral severity score. J Clin Neurophysiol. 2018:35(4):314-24.

31. Bauerschmidt A, Rubinos C, Claassen J. Approach to managing periodic discharges. J Clin Neurophysiol. 2018;35(4):309-13.

32. Lever J, Krzywinski M, Altman N. Classification evaluation. Nat Methods. 2016;13(8):603-4.

33. Ronner HE, Ponten SC, Stam CJ, Uitdehaag BM. Inter-observer variability of the EEG diagnosis of seizures in comatose patients. Seizure. 2009;18(4):257-63.

34. Gaspard N, Hirsch LJ, LaRoche SM, Hahn CD, Westover MB, Critical Care EEG Monitoring Research Consortium. Interrater agreement for Critical Care EEG Terminology. Epilepsia. 2014;55(9):1366-73.

35. Tu B, Young GB, Kokoszka A, Rodriguez-Ruiz A, Varma J, Eerikäinen LM, Assassi N, Mayer SA, Claassen J, Särkelä MOK. Diagnostic accuracy between readers for identifying electrographic seizures in critically ill adults. Epilepsia Open. 2017;2(1):67-75.

36. Jing J, Herlopian A, Karakis I, Ng M, Halford J, Lam A, Maus D, Chan F, Dolatshahi M, Muniz C, et al. Interrater reliability of experts in identifying interictal epileptiform discharges in electroencephalograms. JAMA Neurol. 2020;77(1):49-57.

37. Gururangan K, Parvizi J. Midline and parasagittal seizures are rare in adult patients. Neurocrit Care. 2020;32(1):193-7.

38. Gururangan K, Razavi B, Parvizi J. Diagnostic utility of eight-channel EEG for detecting generalized or hemispheric seizures and rhythmic periodic patterns. Clin Neurophysiol Pract. 2018;3:65-73.

39. Westover MB, Gururangan K, Markert MS, Blond BN, Lai S, Benard S, Bickel S, Hirsch LJ, Parvizi J. Diagnostic value of electroencephalography with ten electrodes in critically ill patients. Neurocrit Care. 2020;33(2):479-90.

40. Drew BJ, Califf RM, Funk M, Kaufman ES, Krucoff MW, Laks MM, Macfarlane PW, Sommargren C, Swiryn S, Van Hare GF. Practice standards for electrocardiographic monitoring in hospital settings: an american heart association scientific statement from the councils on cardiovascular nursing, clinical cardiology, and cardiovascular disease in the young. Circulation. 2004;110(17):2721-46.

41. Amorim E, Williamson CA, Moura LMVR, Shafi MM, Gaspard N, Rosenthal ES, Guanci MM, Rajajee V, Westover MB. Performance of spectrogrambased seizure identification of adult EEGs by critical care nurses and neurophysiologists. J Clin Neurophysiol. 2017;34(4):359-64.

42. Kang JH, Sherill GC, Sinha SR, Swisher CB. A trial of real-time electrographic seizure detection by neuro-ICU nurses using a panel of quantitative EEG trends. Neurocrit Care. 2019:31(2):312-20.

43. Parvizi J, Gururangan K, Razavi B, Chafe C. Detecting silent seizures by their sound. Epilepsia. 2018:59(4):877-84. 